\title{
Traumatic Acinetobacter Baumannii Meningitis Without Neuro surgical Intervention-A Rare Case Report
}

\author{
Suresh. $\mathrm{J}^{1}$, Balaji. ${ }^{1}$, V. Santhosh ${ }^{1}$, M. Nithish Mukunthan ${ }^{1}$, \\ A. L. Shanmuga Priya ${ }^{1}$, Rajaram Manoharan
}

\section{Abstract}

Acute bacterial meningitis is the medical emergency which warrants an early diagnosis and an aggressive therapy. Despite the availability of potent newer antibiotics, the mortality caused by acute meningitis and its complication remains high in India, ranging from $16 \%$ to $32 \%$. The aim of this case report is to present the isolation of Acinetobacter baumannii from cerebrospinal fluid(CSF) of a elderly male following trauma without any neurosurgical intervention and without any evident cerebrospinal fluid otorrhea or rhinorrhea.

Keywords: CSF; Acinetobacter; Colistin; Intraventricular. Introduction

Acinetobacter baumannii is generally considered an opportunistic nosocomial pathogen especially in intensive care unit setting causing infections including bacteremia, pneumonia, ventilator associated pneumonia, meningitis, urinary tract infections and catheter related infection. Emergence of multi drug resistant and extensively drug resistant strains has become a clinical entity of considerable importance resulting in therapeutic dilemma. We report a case of 73 years old man with road traffic accident who developed meningitis in the post operative period without any neurosurgical intervention and without any CSF otorrhea and CSF rhinorrhea.

\section{Case Report}

73-years male Known case of Diabetes mellitus/Systemic hypertension sustained road traffic accident and presented to emergency room. On arrival his Glascow coma scale was E4M5V6. Neuro imaging done which showed lefort II fracture involving right zygomatic process, lateral wall of orbit, anterior and lateral wall of maxillary antra and body of sphenoid sinus. He also had right proximal humerus fracture and right rib fracture $3^{\text {rd }}-6^{\text {th }}$. Neurological examination revealed traumatic optic nerve injury with visual loss. After thorough pre operative assessment he was taken up for open reduction and internal fixation of humerus and zygoma

under general anaesthesia with ultrasound guided interscalene block. He was extubated and observed in Intensive care unit. On III ${ }^{\text {rd }}$ post operative day he had high grade fever with lung signs. Computerised Tomography of chest showed air space disease sequelae of aspiration pneumonitis and lung contusions. Antibiotics were stepped to injection Cefoperazone + sulbactum 3 gram Intravenously twice a day and Clindamycin $300 \mathrm{mg}$ intravenously twice a day. Samples were sent for blood, urine, wound, tracheal culture sensitivity to rule out other foci of sepsis along with inflammatory markers. On $5^{\text {th }}$ post operative day patient had fever spikes with altered sensorium, Clinical examination revealed nuchal rigidity. Repeat Computerised tomography scan of brain done which showed tetra ventricular dilatation with effacement of sulci. After ruling out other causes of metabolic encephalopathy, lumbar puncture done which showed purulent, turbid CSF which was sent for analysis and culture sensitivity. He was empirically started on injection meropenam 2 gram intravenously twice daily and vancomycin 1 gram intravenously twice daily. On $6^{\text {th }}$ post operative day patient became deeply drowsy with increasing respiratory distress for which he was intubated and mechanically ventilated. He was taken up for emergency right frontal external ventricular drain insertion. Magnetic resonance imaging of brain done which showed severe signs of meningitis. CSF culture showed acinetobacter baumannii

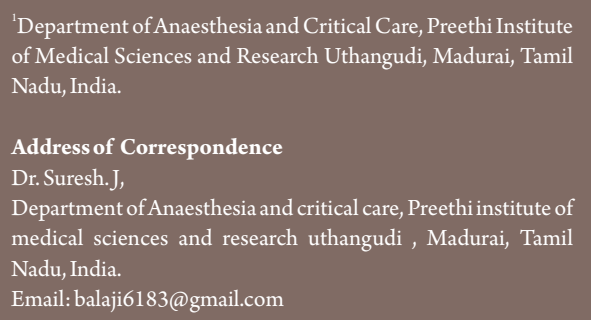

${ }^{1}$ Department of Anaesthesia and Critical Care, Preethi Institute of Medical Sciences and Research Uthangudi, Madurai, Tamil Nadu, India.

Address of Correspondence

Dr.Suresh.J,

Nadu, India.

Email:balaji6183@gmail.com



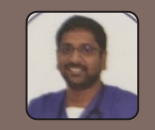

Dr. T. Balaji

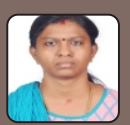

Dr. Shanmuga Priya



Dr. V. Santhosh

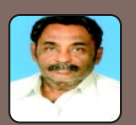

Dr. Rajaram Manoharan

2021 (c) Journal of Anaesthesia and Critical Care Case Reports| Available on www.jaccr.com | ISSN 2454-7174| DOI: 10.13107/jaccr.2021.v07i01.170

This is an Open Access article distributed under the terms of the Creative Commons Attribution Non-Commercial License (http://creativecommons.org/licenses/by-nc/3.0)

which permits unrestricted non-commercial use, distribution, and reproduction in any medium, provided the original work is properly cited.

22 | Journal of Anaesthesia and Critical Care Case Reports | Volume 7 | Issue 1 | January-April 2021 | Page 22-25 


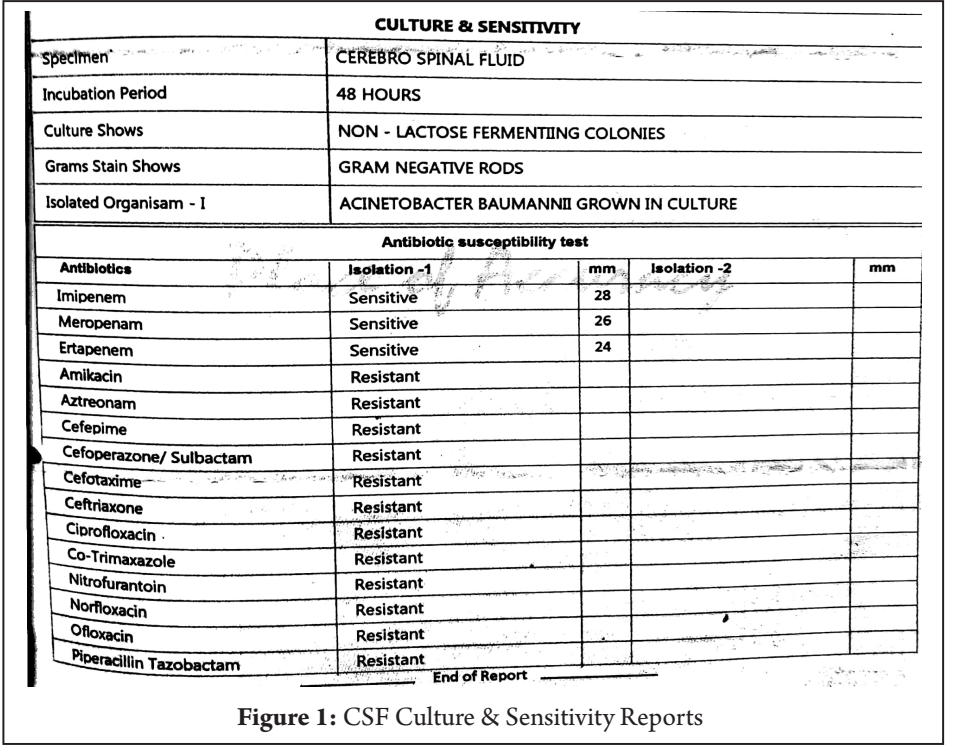

which is a multi drug resistant strain with susceptibility to meropenam, imipenam and colistin. He was then started on Colistin 6 million units intravenously stat and 2 million units thrice a day and meropenam was continued. In view of persistent fever spikes with no significant improvement in sensorium he was started on intraventricular colistin one lakh units per day. Tracheostomy was done on $13^{\text {th }}$ post operative day in view of need for prolonged ventilator support. Antiepileptic drugs were stepped up for recurrent focal seizures. CSF analysis done which showed mild reduction in total counts and external ventricular drain was removed. Inspite of aggressive efforts patient deteriorated with septic shock and eventually succumbed.

\section{Discussion}

Acinetobacter baumannii is a nosocomial pathogen of increasing clinical importance [1]. Among the outbreaks of infection associated with acinetobacter baumannii, nosocomial pneumonia is the most common clinical manifestation [2].

Specific concerns has been the increasing prevalence of multidrug and pandrug resistance strains.Acinetobacter baumannii is an exceedingly rare cause of community acquired meningitis but is an important pathogen associated

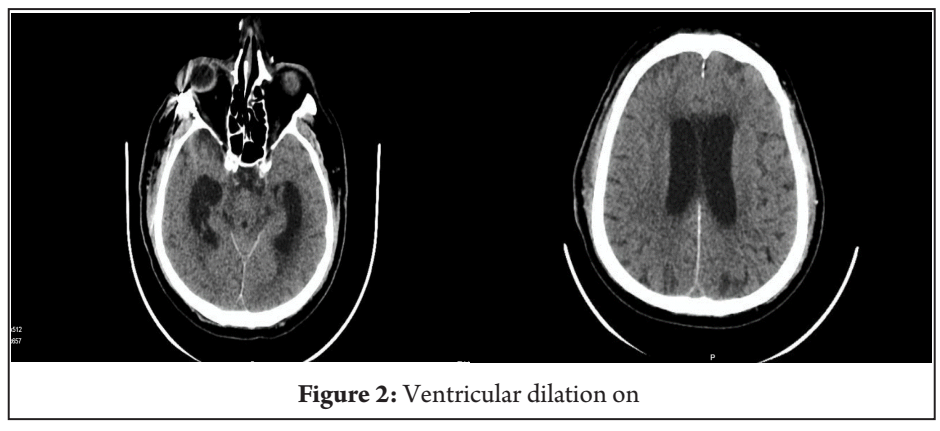

with post neurosurgical meningitis. Literature reveals that acinetobacter species account for just $0.2 \%$ of community acquired meningitis in adults [3]. It was also a rare cause of community acquired bacterial meningitis in children [4]. Acinetobacter species accounts for $3.6 \%$ of hospital acquired meningitis [5]. Acinetobacter meningitis typically occurs following neurosurgery. Patients with CSF leakage, concomitant incision infection, prolonged duration of surgery, surgery that enters a sinus, prolonged external ventricular drain, and need for repeat surgery are at increased risk of post neurosurgical meningitis [6].

Neurosurgical procedures commonly associated with postoperative acinetobacter species meningitis include craniotomy, insertion or exchange of external ventricular drain, Ventriculo peritoneal shunt and insertion of lumbar drain. The median time to develop acinetobacter meningitis after a neurosurgical procedure is 12 days (range 1-40 days) [7].

All cause mortality from acinetobacter meningitis ranges from $15 \%-71 \%$ [8]. Highest mortality rates have been observed in neonates and in centers where carbapenem resistance strains seen [9].

Acinetobacter is a gram negative coccobacillus that are non motile, catalase positive, oxidase negative [10]. Acinetobacter baumannii is the most resistant of the genospecies, other strains that have been associated with disease include acinetobacter johnsonii, acinetobacter iwoffi. Acinetobacter baumannii is one of the ESCAPE organisms, a group of clinically important organism that have potential for substantial antimicrobial resistance [11]. Acinetobacter has the ability to develop resistance through several mechanisms. Frequently observed resistance mechanisms in nosocomial strains include production of beta lactamases, alterations in cell wall channels and efflux pumps [12].

Risk factors for colonization or infection with resistant strains of acinetobacter include prior colonization with methicillin resistant staphylococcus aureus (MRSA), prior beta lactam and fluoroquinolone usage, bedridden status, recent surgery, mechanical ventilation, hemodialysis and malignancy [13]. Multidrug resistant acinetobacter means, the isolate is susceptible to atleast one agent in three or more antibiotic classes. Extensively drug resistant means isolate is non susceptible to at least one agent in all but two or fewer antibiotic classes as proposed by Joint initiative by European and US Centers for Disease Control. Patient with resistant strains appears to have higher mortality rate than infection with susceptible strains [14]. Most patients with acinetobacter meningitis present with fever, meningeal signs and seizures. CSF typically demonstrates pleocytosis with neutrophilic predominance, increased protein levels and low 
CSF-to-serum glucose ratio [15].

When infections are caused by antibiotic susceptible isolates, there are many therapeutic options including a broad spectrum cephalosporin (ceftazidime or cefepime), a combination beta lactam- beta lactamase inhibitor (includes sulbactum), or a carbapenem. Carbapenems are highly bactericidal against susceptible strains of acinetobacter [16]. Combination antimicrobial therapy is frequently used to increase the likelihood of adequate empiric antibiotic coverage before drug susceptibility testing results are known to decrease risk of emergent resistance and to improve outcomes in multi drug resistance infections.

Carbapenems most reliably enter into the CSF particularly when meningeal inflammation is minimal [17]. Meropenem is the most appropriate choice of carbapenem. For central nervous system infection the dose should be $2 \mathrm{~g}$ Iv every eight hour. High dose imipenem was associated with increased incidence of seizures. Polymyxins has been used in success for carbapenem resistant isolates. There is moderate penetration of inflamed meninges and spinal fluid levels reach approximately $25 \%$ of serum levels with intravenous colistin [18]. For this reason, central nervous system infections with drug resistant acinetobacter warrants use of intrathecal or intraventricular colistin, The dose range of intraventricular or intrathecal colistin therapy has varied widely from $0.75-7.5 \mathrm{mg}$ colistin base activity (25,000-2,50,000 IU colistimethate sodium) total daily dose(19). Complications of intraventicular colistin includes aseptic chemical meningitis, ventriculitis. Dose reduction is needed as CSF white cell count increases. The treatment of acinetobacter meningitis is usually atleast 3 weeks. The response should be assessed clinically and with repeat CSF cultures.
Conclusion

Acinetobacter species are gaining clinical importance with emergence of extended resistance to even newer antimicrobials. They also have immense potential to cause nosocomial outbreaks. We report this rare case because of isolation of acinetobacter baumannii from CSF of a patient in the postoperative period without any neurosurgical intervention and without any CSF otorrhea and CSF rhinorrhea. Thus to decrease the spread of acinetobacter infections, to reduce the mortality and morbidity of patients and to reduce the emergence of multidrug resistant acinetobacter, it is important to promote rationale use of antimicrobials. Hand hygiene and barrier nursing are equally important to keep the infection in check.

\section{Clinical Message}

This case highlights the issues involved in treating infections caused by multi drug resistant and pan drug resistant acinetobacter meningitis. Meningitis should also be suspected in patients with base of skull fractures with new onset fever or any unexplained deterioration in neurological status even though there is neuro surgical intervention or evident CSF rhinorrhea or otorrhea. Early diagnosis and appropriate antibiotic are cornerstones of reducing mortality and morbidity.

\section{References}

1. Karageorgopoulos DE, Falagas ME. Current control and treatment of multidrug-resistant Acinetobacter baumannii infections. Lancet Infect Dis. 2008;8:751-62.[PubMed] [Google Scholar

2. Perez F, Hujer AM, Hujer KM, Decker BK, Rather PN, Bonomo RA. Global challenge of multidrug-resistant Acinetobacter baumannii. Antimicrob Agents Chemother. 2007;51:3471-84. [PMC free article] [PubMed] [Google Scholar]

3. Hussein AS, Shafran SD. Acute bacterial meningitis in adults. A 12-year review. Medicine (Baltimore) 2000;79:360-68. [PubMed] [Google Scholar]

4. Husain E, Chawla R, Dobson S, Dele Davies H. Epidemiology and outcome of bacterial meningitis in Canadian children: 1998-1999. Clin Invest Med. 2006;29:131-35. [PubMed] [Google Scholar]

5. Weisfelt $M$, van de Beek D, Spanjaard L, de Gans J. Nosocomial bacterial meningitis in adults: a prospective series of 50 cases. J Hosp Infect. 2007;66:71-78. [PubMed] [Google Scholar]
6. Korinek AM, Baugnon T, Golmard JL, van Effenterre R, Coriat P, Puybasset L. Risk factors for adult nosocomial meningitis after craniotomy: role of antibiotic prophylaxis. Neurosurgery. 2006;59:126-33. discussion 126-33. [PubMed] [Google Scholar]

7. Siegman-Igra Y, Bar-Yosef S, Gorea A, Avram J. Nosocomial acinetobacter meningitis secondary to invasive procedures: report of 25 cases and review. Clin InfectDis. 1993;17:843-49. [PubMed] [Google Scholar]

8. Chen SF, Chang WN, $\mathrm{Lu} C \mathrm{CH}$, et al. Adult acinetobacter meningitis and its comparison with non-acinetobacter gram-negative bacterial meningitis. Acta Neurol Taiwan.2005;14:131-37. [PubMed] [Google Scholar]

9. Huttova M, Freybergh PF, Rudinsky B, et al. Postsurgical meningitis caused by Acinetobacter baumannii associated with high mortality. Neuro Endocrinol Lett.2007;28 (suppl2):15-16. [PubMed] [Google Scholar]

10. Juni E. Interspecies transformation of Acinetobacter: genetic evidence for a ubiquitous genus. J Bacteriol 1972; 112:917 
11. Rice LB. Federal funding for the study of antimicrobial resistance in nosocomial pathogens: no ESKAPE. J Infect Dis 2008; 197:1079.

12. Mugnier P, Poirel L, Pitout M, Nordmann P. Carbapenem-resistant and OXA-23-producing Acinetobacter baumannii isolates in the United Arab Emirates. Clin Microbiol Infect 2008; 14:879.

13. Tacconelli E, Cataldo MA, De Pascale G, et al. Prediction models to identify hospitalized patients at risk of being colonized or infected with multidrugresistant Acinetobacter baumannii calcoaceticus complex. J Antimicrob Chemother 2008; 62:1130.

14. Lemos EV, de la Hoz FP, Einarson TR, et al. Carbapenem resistance and mortality in patients with Acinetobacter baumannii infection: systematic review and meta-analysis. Clin Microbiol Infect 2014; 20:416.

15. Rodríguez Guardado A, Maradona JA, Asensi V, et al. [Postsurgical meningitis caused by Acinetobacter baumannii: study of 22 cases and review of the literature]. Rev Clin Esp 2001; 201:497.
16. Fishbain J, Peleg AY. Treatment of Acinetobacter infections. Clin Infect Dis 2010;51:79.

17. Kim BN, Peleg AY, Lodise TP, et al. Management of meningitis due to antibiotic-resistant Acinetobacter species. Lancet Infect Dis 2009; 9:245.

18. Jiménez-Mejías ME, Pichardo-Guerrero C, Márquez-Rivas FJ, et al. Cerebrospinal fluid penetration and pharmacokinetic/pharmacodynamic parameters of intravenously administered colistin in a case of multidrugresistant Acinetobacter baumannii meningitis. Eur J Clin Microbiol Infect Dis 2002; 21:212.

19. Ng J, Gosbell IB, Kelly JA, et al. Cure of multiresistant Acinetobacter baumannii central nervous system infections with intraventricular or intrathecal colistin: case series and literature review. J Antimicrob Chemother 2006; 58:1078.

Acknowledgments: We like to thank professor Dr.Sivakumar.R Chairman and Dr.Hema Sivakumar MD, Preethi Institute of Medical sciences and Research for motivating us.

\section{Conflict of Interest: Nil} Source of Support: None

\section{How to Cite this Article}

Suresh J, Balaji T, Santhosh V, Mukunthan MN, Priya ALS, Manoharan R | Traumatic Acinetobacter Baumannii Meningitis Without Neuro surgical Intervention- A Rare Case Report | Journal of Anaesthesia and Critical Care Case Reports | January-April 2021; 7(1): 22-25. 